\title{
A transit-signal methodology for studying reaction time ${ }^{\prime}$
}

\author{
RO B E R T G O T T S D A NKER, \\ UNIVERSITY OF CALIFORNIA, SANTA \\ BARBARA, Santa Barbara, Califormia \\ 93106
}

\section{A new general method has been devised for measuring reaction time, which combines the traditional transit and signal methods. Some applications are described. Advantages over the traditional procedures are considered.}

A new general method has been devised for studying RT (reaction time) that combines the traditional transit and the signal methods. In the transit method, $S$ is instructed to respond when a moving target point crosses a reference line. In the signal method, $\mathrm{S}$ responds to the onset or offset of a stimulus of whatever nature $E$ wishes to use. An example of the combined, or transit-signal, method is shown in Fig. 1. The disk rotates slowly at a constant rate. The lamp, L, may flash at any instant while the white target sector moves past the reference line, $R$. The task for $S$ is to respond to the onset of the light flash. Of course, the signal needn't be visual or the task limited to the simple a-reaction, although it is possible that the moving target may be distracting when discriminations must be made. This method, considered more generally, can be shown to possess most of the separate advantages of the transit and the signal methods but not their liabilities and, further, to lead to procedures that are powerful in delineating the mode of operation of the informational variables that influence response latency.

The transit and signal methods would appear to be the only techniques possible for studying RT. Both were employed in the pioneer study of RT-or "absolute personal equation"-by the astronomer, Mitchel (1858). However, the transit method has rarely been used, most probably because of the realization that $S$ tends to synchronize his response with the transit rather than to respond to it per se. Even so, it was found early that catch trials provide an adequate remedy (Günther, 1911). In any event, the method does have serious limitations. It is restricted to simple visual reactions (if the rather unpromising auditory and cutaneous analogues are excluded) and to relatively meager possibilities for interesting experimental manipulation.

The advantages of the signal method over the transit method are many. The signal may be of any modality and quality

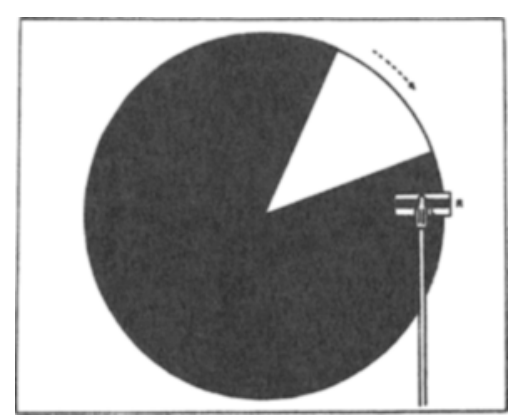

Fig. 1. The transit-signal display.

desired. Choices and identifications of all kinds may be studied. It is no wonder that this has been the preferred method. Yet, the determination of a minimum value for the simple or a-reaction is subject to exactly the same difficulties due to synchronizing as the transit method, and the best solution again appears to be the use of catch trials. In fact, the transit method is somewhat superior in this respect as it avoids the complications of timekeeping inaccuracy. Because of the possibility of synchronized responses, the obvious question of the optimum constant FP (foreperiod between warning signal and response signal) has not even yet been answered. For example, Klemmer (1956) reported that the minimum RT at the shortest constant FP used was $250 \mathrm{msec}$. However, $\mathrm{S}$ should easily be able to make a response to the warning signal since there are no catch trials, taking care that his response will always be at least a little later than the response signal. Long constant FPs are necessary if this strategy is to be frustrated. Here, advantage is taken of S's (unassessed) inadequacy as a timekeeper. While this procedure does not lead to minimum RTs, it does permit the comparison of experimental conditions. Further, as Klemmer has shown (1957), a measure of timekeeping accuracy may, in fact, be obtained by giving $S$ the task of synchronizing on another set of trials so that the contribution of this factor may be evaluated. A problem here is that $S$ must "learn" and remember the interval from one trial to another, whether engaged in the RT or the synchronization task. Better experimental control would be to use a countdown technique with several presentations of the interval, as Cowan and Monroe (1968) and Symington (1969) have done. Only short-term rhythmic memory is involved.

Another way of preventing synchronization is to use varied FPs.
However, the problems of learning and memory are much more serious than with a long, constant FP. Also, the determination of how well S has "internalized" the range is not obviously soluble. Obviously, no countdown method may be employed. It should be noted that the method succeeds only because of S's faulty learning or memory of the instants at which the signal may occur. Otherwise, he would be able to synchronize at the end of the interval. The varied FP method that arose out of the need to prevent synchronization has itself become the object of study with the rise of interest in informational variables in RT. The problem becomes that of the relation of objective time uncertainty to RT (e.g., Klemmer, 1956, 1957; Karlin, 1959, 1966; Drazin, 1961). However, use of varied FPs does not seem adequate for this purpose for three reasons. First, there is no separation of objective time uncertainty and timekeeping inaccuracy. As values in ranges become longer, timekeeping becomes poorer. Second, different ranges can be compared only if the midpoint value is long, since a wide range cannot have a low midpoint. If the midpoint value becomes long enough, it is likely that the effects of range will be minimized. Third, as no signal has yet occurred on a trial, the momentary probability increases until it becomes unity. The resulting anticipation effect may be modified by unequal distributions of signal probability during the range (Karlin, 1966). Yet, it can only be eliminated by use of catch trials, which the method of varied FPs was devised to circumvent.

This leads to the final technique, by which synchronizations may be prevented, and which has been devised specifically to deal with the question of temporal uncertainty: nonaging FPs (Nickerson \& Burnham, 1969). In this method, momentary probability of the signal remains constant, being expressed by the probability, $p$, in relation to an epoch of duration, T. Still, there are some problems that are not essentially different from those with different FP ranges. First, the method depends upon the abstraction of some equivalent of $p$ and $T$ from experience with past FPs. This would seem even more difficult for $S$ to learn and remember than a range of values, and the assessment of how well it has been done even more puzzling. Second, with a low value of $p$ or a high value of $T$, curtailed distributions must be used to make the method practical. Otherwise, there would be some very long waits indeed. The 
Fig. 2. The arrangement used in preliminary studies. $M$ is the Slo-Syn motor used for rotating the aisk.

problem here (and also when catch trials are used with a range of FPs) is that while momentary probability remains constant, the probability that there will be no signal at all on the trial increases from the beginning of the range. Perhaps this probability is the more important one for RT.

We may now consider some applications of the transit-signal method and consider, with each example, how the difficulties of the separate transit and signal methods are overcome. First, a general definition of the transit-signal method is needed. It is the RT procedure in which $S$ is informed of when a signal can occur by the coincidence of a moving target and a stationary reference line or zone.

\section{Event Uncertainty}

In this application, the rotating target is reduced to line width. Over a block of trials, the lamp that is even with the reference line is flashed on a predetermined proportion of crossings. The function may then be found between RT and probability that the signal will occur. An advantage may be seen over the signal method for studying the problem. With that method, a constant FP is used since the intention is to find the effect of event uncertainty without the confounding influence of time uncertainty. The main problem is that, even with a short FP, there is considerable timekeeping inaccuracy. Symington (1969) found the SD of synchronizations for a $1 \cdot \mathrm{sec}$ constant FP to be about $125 \mathrm{msec}$. Of course, an FP shorter than $1 \mathrm{sec}$ would give a smaller timekeeping error, but any value much less than $1 / 2 \mathrm{sec}$ brings about the possibility of psychological refractoriness. Hence, the lowest possible SD of synchronization would be at least $50 \mathrm{msec}$. By way of contrast, Slater-Hammel (1960) found synchronization of transits to have an SD of only $19 \mathrm{msec}$, no knowledge of results being given to $S$.

Another problem of the signal method is that, with lower probability, $S$ experiences the FP less often and may thus have a poorer time estimation of when the signal will occur. Gordon (1969) may have solved this problem by increasing the trial density proportionally to the decrease in probability. However, $S$ still has unequal proportions of times of experiencing the FP with varying probabilities of signal occurrence. It cannot be assumed that this variable will have no effect on time estimation. In contrast, there seems no reason why estimation of moment of

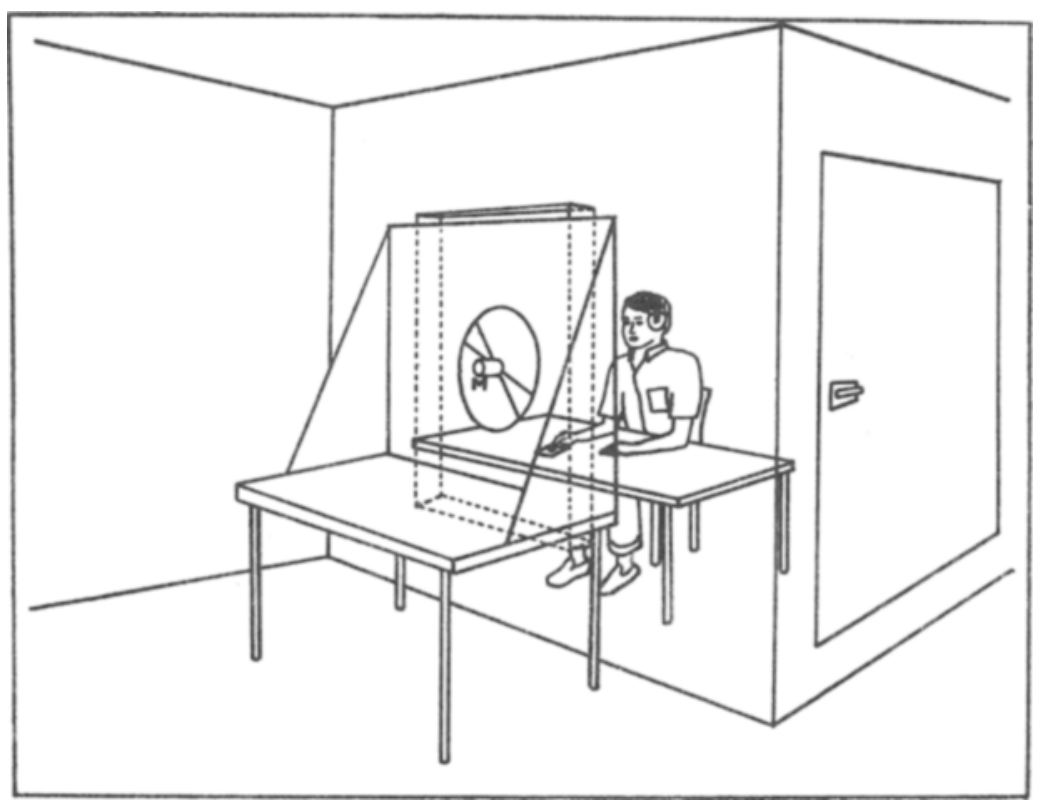

coincidence should be affected by maximum preparation for a signal may be probability of signal occurrence in the studied by putting line-width sectors transit-signal method. Preliminary results various distances apart on the disk. The indicate much less increase in RT as a probability of occurrence should be varied consequence of low probability with the since it is likely an important factor in signal-transit method than with the signal method (Gottsdanker, 1968). The way to study this problem with the pure transit method would be for the target to stop or disappear suddenly, just before coincidence with the reference line (Günther, 1911; Hammer, 1913). The chief deficiency would be restriction to this one type of visual situation.

\section{Time Uncertainty}

The arrangement shown in Fig. 1 is used. In preliminary testing, the signal could occur at any one of nine instants as the white sector traversed the reference line. Amount of objective time uncertainty is controlled by width of the sector. It is seen that, unlike the case for the signal method (with warning signal), there is no reliance upon memory of the range of FPs. The very small error of synchronization of a transit essentially eliminates timekeeping inaccuracy. Also, the confounding variable of value of midrange is avoided. A preliminary comparison with the signal method reveals a more pronounced reduction in RT for signals that occur at the end of the range (Gottsdanker, 1968). It is obvious that this problem cannot be investigated with the pure transit method.

\section{Other Applications}

The preceding examples have already had preliminary testing. Plans for future research are presented below.

(1) The length of time required to reach preparation. It is expected that RT will diminish, up to a point, as intersector distance is increased.

(2) A direct method of controlling (rather than eliminating) timekeeping will be studied by placing a mask, behind which the target line-width sector disappears, before the reference line. It is expected that as the required prediction span is made longer, the less accurate will be the judgment of when the signal will occur. A test will be made of S's ability to synchronize and to make RT responses under this condition. A much better control of time-keeping accuracy should result than by the use of remembered FPs (e.g., Klemmer, 1957). RT may then be related to timekeeping accuracy.

(3) Psychological refractoriness, the increase in RT because of a closely preceding signal, has not been exhibited consistently in conditions in which $S$ is unprepared for the first signal (e.g., John, 1964; Bertelson, 1967). This effect may be studied systematically by means of masks of different widths.

\section{APPARATUS}

Figure 2 shows the way in which the disk has been employed. In the interest of putting everything into one picture, the visual signal, the lighting of a neon lamp, is indicated, although $S$ is depicted as wearing headphones to receive an auditory signal. The disk is made of plywood, painted black, and is 18 in. in diam. The motor is a 
Slo Syn Type HS50 that moves in discrete steps of $1.8 \mathrm{deg}$ or 200 for a complete revolution. It was driven by a $25 / \mathrm{sec}$ train of pulses generated by a system of logic units, giving a complete revolution each $8 \mathrm{sec}$.

At the present time, there is a good deal that is makeshift and arbitrary about the arrangement. A half-reflecting mirror could be used to advantage for positioning the reference line and signal light. Times during which $S$ sees the display could be controlled more effectively by presenting him with a television picture rather than with the direct display. This would also eliminate the necessity of having $S$ watch the initial revolution of the disk after stopping, during which time it shudders considerably. The rate of 25 steps per second was chosen as the slowest that allows good apparent motion. Faster rates should also be studied. Moreover, it is difficult to obtain smooth performance of the motor with low pulse rates. This may be remedied by use of gear reduction and a much higher pulse rate. However, it seems a good idea to stay with the simple mechanical disk rather than to go over to more sophisticated methods. There is great advantage in being able to change the experimental condition simply by painting a new sector or two on the disk or perhaps a reference zone rather than a line.
EVALUATION

The new method seems useful largely because it utilizes direct perception of motion and is thereby free from the dependence on trial-to-trial memory, a characteristic of the signal method. It is quite possible that many of the findings obtained to date on RT have been influenced heavily by peculiarities of the signal method. Such variables as objective time uncertainty and timekeeping inaccuracy, which are hopelessly intertwined in the signal method, can be controlled independently by the transit-signal method. Certainly, the examples shown are merely a start of the applications of the general method.

\section{REFERENCES}

BERTELSON, P. The time course of preparation. Quarterly Journal of Experimenta Psychology, 1967, 19, 272-279.

COWAN, T. M., \& MONROE, B. D. Null stimulus reaction time. Paper presented at the meeting of the Psychonomic Society, 1968.

DRAZIN, D. H. Effects of foreperiod, foreperiod variability, and probability of stimulus occurrence on simple reaction time. Journal of Experimental Psychology, 1961, 62, 43-50.

GORDON, I. E. Stimulus probability and simple reaction time. Nature, $1967,215,895-896$.

GOTTSDANKER, R. Reaction time with near-perfect timekeeping. Paper presented at the meeting of the Psychonomic Society, 1968.

GÜNTHER, F. Reaktionsversuche bei
Durchgangsbeobachtungen. Psychologische Studien, 1911, 7, 229-285.

HAMMER, A. Untersuchung der Hemmung einer vorbereiteten Willenshandlung. Psychologische Studien, 1913, 9, 321-365.

JOHN, I. D. The role of extraneous stimuli in responsiveness to signal: Refractoriness or facilitation. Australian Journal of Psychology, 1964, 16, 87-96.

KARLIN, L. Reaction time as a function of foreperiod duration and variability. Journal of Experimental Psychology, 1959, 58, 185-191.

KARLIN, L. Development of readiness to respond during short foreperiods. Journal of Experimental Psychology, 1966, 72, 505-509.

KLEMMER, E. T. Time uncertainty in simple reaction time. Journal of Experimental Psychology, 1956, 51, 179-184.

KLEMMER, E. T. Simple reaction time as a function of time uncertainty. Journal of Experimental Psychology, 1957, 54, 195-200. MITCHEL, O. M. On personal equation. Journal of Franklin Institute, 1858, 66, 349-352.

NICKERSON, R. S., \& BURNHAM, D. W. Response times with nonaging foreperiods. Journal of Experimental Psychology, 1969, 79, 452-457.

SLATER-HAMMEL, A. T. Reliability, accuracy and refractoriness of a transit reaction. Research Quarterly, 1960, 31, 217-228.

SYMINGTON, L. E. Reaction time and timekeeping accuracy. PhD dissertation, University of California, Santa Barbara, 1969.

NOTE

1. This work was supported by Research Grant MH 10447 from the National Institute of Mental Health and by a grant from the Research Committee of the University of Califomia. 